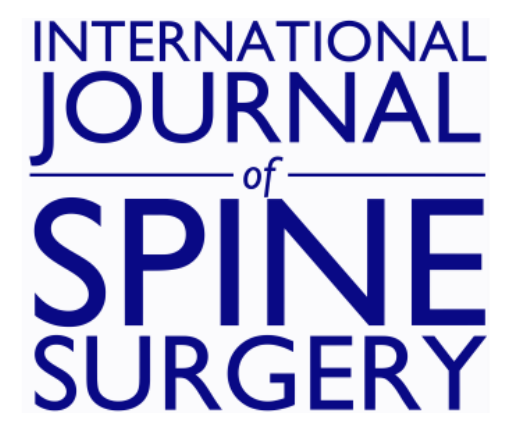

\title{
Variability in Flexion Extension Radiographs of the Lumbar Spine: A Comparison of Uncontrolled and Controlled Bending
}

Boyle Cheng, Anthony E. Castellvi, Reginald J. Davis, David C. Lee, Morgan P. Lorio, Richard E. Prostko and Chip Wade

Int J Spine Surg 2016, 10 ()

doi: https://doi.org/10.14444/3020

http://ijssurgery.com/content/10/20

This information is current as of April 25, 2023.

Email Alerts Receive free email-alerts when new articles cite this article. Sign up at:

http://ijssurgery.com/alerts

The International Journal of Soding Surgerght 2397 Waterbury Circle, Suite 1,

Aurora, IL 60504, Phone: +1-630-375-1432 


\section{Variability in Flexion Extension Radiographs of the Lumbar Spine: A Comparison of Uncontrolled and Controlled Bending}

Boyle Cheng, Ph.D., 1 Anthony E. Castellvi, MD, ${ }^{2}$ Reginald J. Davis, MD, ${ }^{3}$ David C. Lee, MD, ${ }^{4}$ Morgan P. Lorio, MD, 5 Richard E. Prostko, MD, ${ }^{6}$ Chip Wade, Ph.D. ${ }^{6}$

${ }^{1}$ Department of Neurosurgery, Drexel University College of Medicine, Pittsburgh, PA, ${ }^{2}$ Florida Orthopedic Institute, Tampa, FL, ${ }^{3}$ Department of Neurosurgery, Greater Baltimore Medical Center, Baltimore, MD, ${ }^{4}$ Southern Neurologic and Spinal Institute, Hattiesburg MS, 5 Neuro-Spine Solutions, Bristol, TN, ${ }^{6}$ Department of Industrial and Systems Engineering, Auburn University, Auburn, AL

\section{Abstract}

Background

While low back pain is one of the most prevalent, if not the most prevalent reasons for visits to physicians, a majority of patients with low back pain cannot be given a definitive diagnosis. While there have been substantial advances in imaging technologies over the past 30 years, relatively little has changed in the methodologies for evaluating functionality of the lumbar spine. The current standard of care for function assessment of the lumbar spine focuses on uncontrolled patient directed motion which results in increased inter-patient variability. Recent advancements in functional lumbar spine testing utilize controlled bending and computerized imaging evaluation.

Purpose

To compare the measurement variability of lumbar spine motion when diagnosed using measurements of intervertebral motion taken from standard bending flexion/extension radiographs (FE) between uncontrolled and controlled motion.

\section{Study Design}

One-hundred nine patients (57 asymptomatic, 52 symptomatic) were consented in the prospective investigation. The research was designed to compare studies involving FE to controlled motion bending radiographs using the Vertebral Motion Analysis (VMA), (Ortho Kinematics, Inc) within the same patient. Each patient agreed to undergo fluoroscopic still imaging to capture FE data and to undergo cine fluoroscopic imaging to capture VMA data.

\section{Outcome Measures}

Measurement variability was determined by the mean and standard deviation of intervertebral rotation when evaluated by 5 independent observers evaluating each of the 109 patients FE and VMA. The resulting standard deviation of the intervertebral rotation determinations was used as the measure of variability.

\section{Methods}

The VMA measurements for assessing intervertebral motion were characterized by the use of: (1) a handling device that assists patients through a standard arc of lumbar bending in both an upright and recumbent posture (70 degree flexion/extension arcs; 60 degree left/right bending arcs); (2) video fluoroscopy imaging of the lumbar spine during bending (capturing images at 8 frames per second); and (3) image processing software capable of automatic frame-to-frame registration and tracking of vertebral bodies across the sequence of video-fluoroscopic images to derive measurements of intervertebral rotation and translation. The FE data were assessed from voluntary bending by the patient.

Results

There was statistical greater measurement variability in intervertebral rotation in FE when compared to VMA (both standing and lying). When comparing measurement variability between FE and VMA, results indicate between a $26 \%$ to $46 \%$ decrease in measurement variability under VMA compared to FE. These findings are consistent across asymptomatic and symptomatic patients. 
Conclusions

The current standard of care for functional testing of the lumbar spine utilizes uncontrolled FE with a manual data evaluation process. Recent developments in using computerized imaging processes has improved, however there remains variability in patient bending due to the self-selected rate and position of the bending. VMA results in a significant reduction in measurement variability of intervertebral rotation measurements.

KEYWORDS: LUMBAR SPINE, FLEXION/EXTENSION RADIOGRAPHS, VERTEBRAL MOTION ANALYSIS (VMA), CONTROLLED MOTION BENDING, VARIABILITY VOLUME 10 ARTICLE 20 DOI: 10.14444/3020

\section{Introduction}

Low back pain (LBP) affects approximately half of all adults in the United States annually and is one of the most prevalent if not the most prevalent reasons for visits to physicians. ${ }^{1}$ The cause of LBP is often unclear, the correspondence between symptom and anatomical findings is low, and up to $85 \%$ of patients with low back pain cannot be given a definitive diagnosis. ${ }^{2}$ Given the corollaries between the epidemiology and the ineffective/incomplete diagnosis modalities, it's not surprising that the clinical standard of care for performing functional testing of the spine was introduced more than a half century ago with minimal advancement in diagnostic methodologies since.

Drawing a parallel between non-specific back pain and non-specific chest pain there are similarities and available diagnostic test to assess the anatomy in the spine (i.e. $x$-rays: bone, MRI: soft tissues) as well as the spine (i.e. $x$-rays: tissue features, CT: vasculature). However, there is a stark contrast in assessing function. The cardiovascular community enjoys a battery of functional test (i.e. Angiography, EKG, stress testing, Echocardiography, others) which results in successful treatment rate in addressing nonspecific chest pain. Other systems have similar diagnostic breadth; the endocrine system is complex and diverse; as such clinicians often conduct a series of dynamic functional tests to pinpoint exactly where the disorder lies. Multitudes of dynamic test for endocrine function exist across a vast range of systems from the adrenal to calcium and bone metabolism function, resulting in similar diagnostic success rates as the cardiovascular community. However, there has yet to be an accepted definitive functional diagnostic test for spine; which likely contributes to a far less successful rate of addressing non-specific back pain.
While there have been substantial advances in imaging technologies over the past 30 years with the utilization of imaging procedures such as computed tomograghy (CT) scans and Magnetic Resonance imaging (MRI); relatively little has changed in the methodologies for evaluating functionality of the lumbar spine. ${ }^{3}$ The current method for conducting functional testing of the spine (flexion/extension and lateral bending) involves capturing standard bending radiograpghs (FE) of the spine as the patient bends, and then holds their spine fixed in the extreme motion in either the sagittal (flexion/extension) or coronal (lateral bending) planes. ${ }^{4,5}$

In recent years, there have been improvements to the methodologies in interpreting the FE. Manual measurements are performed by sketching lines or superimposing a measurement grid on physical films on a light board or computer based system..${ }^{6,7}$ Computerassisted manual measurements are performed by using software to manually construct lines between vertebral landmarks and utilizing the computer software to provide measurement data. ${ }^{8,9,10}$ Automated (semi) computerized measurements are performed in digital radiographs by computer software applying image processing. ${ }^{8,910}$ Previous studies have documented the improvement in precision, sensitivity and reliability of measurements when applying computerized measurements techniques. ${ }^{11-15}$

The causes and effects of measurement variability are fairly understood and can be addressed through computerized measurement techniques, whereas inter-patient bending variability in $\mathrm{FE}$ is less obvious in the current clinical methodologies. FE are uncontrolled and the range of motion through the flexion/ extension arc is driven by the patient. ${ }^{16,17}$ Given patients select the rate and range in which they bend, there is a level of inter-patient and intra-patient variability. There is a foundation of clinical and scientific 
literature which suggest bending radiographs measured throughout a range of continuous controlled motion (VMA) assessing intervertebral motion with the use of a handling device that assists patients through a standard arc of lumbar bending reduces patient driven variability in bending. ${ }^{3,15,18-20}$

The efficacy of diagnostic imaging in patients exhibiting LBP is an important factor in today's health care environment.While there have been improvements to measurement techniques and diagnostic imaging modalities, little has changed in the functional testing of the spine. Recently, the validation of VMA coupled with automated computerized measurement software, has provided an alternative to the ruler and protractor method of the 1940's for evaluating function spine characteristics. The purpose of the current study was to compare the measurement variability of lumbar spine motion when diagnosed using measurements of intervertebral motion taken from FE and VMA.

\section{Materials and Methods}

Patients

One hundred and nine patients (57 asymptomatic and 52 symptomatic) were enrolled in a 6 site (Austin, TX; Baltimore, MD; Bristol, TN; Los Angeles, CA; Pittsburgh, PA; Tampa, FL) diagnostic investigation comparing the classification of patients using FE (Picture 1) to that using the VMA (Picture 2). The VMA were conducted via vertebral motion analysis (VMA) (Ortho Kinematics, Inc; Austin, TX), a method of assessing intervertebral motion that involves device-assisted bending, fluoroscopy imaging, and image processing software.

The patients were enrolled if they: (1) met the inclusion/exclusion criteria, (2) were willing to participate in the study, and (3) offered enrollment by study site staff. The following exclusion criteria were placed for enrollment in the study: morbidly obese (BMI > 40) or incapable of providing sufficient contrast as evidenced by the initial study films; inability to accurately complete the study documentation; claustrophobic; pregnancy or anticipation of becoming pregnant in 24 months; height is greater than 76 inches (Table 1).

\section{Equipment}

The VMA system assessed intervertebral motion through the use of three distinct processes: (1) a patient handling device that assists patients through a prescribe-able arc of lumbar bending in both an upright (Picture 2) and recumbent (Picture 3) posture (flexion/extension arcs were 70 and 60 degrees as measured by the rotation of the VMA for upright and recumbent, respectively); (2) video fluoroscopy imaging of the lumbar spine during bending (capturing images at 8 frames per second) utilizing a standard 12-inch surgical C-Arm (OEC 9800 Radiographs, General Electric, Fairfield, CT, USA; 2006 Phillips BV Pulsera, Andover, MA, USA); (3) console mounted computer equipped with data acquisition hardware (Accustream Express As205A, Foresight Imaging, Chelmsford, MA, USA) to digitize fluoroscopic video signals and (4) proprietary image processing software capable of assisting the user in the semi-automatic frame-to-frame registration and tracking of vertebral bodies across the sequence of video-fluoroscopic images to derive measurements of intervertebral rotation and translation (Ortho Kinematics, Austin, TX, USA). This methodology is consistent over studies utilizing the VAM technology. ${ }^{11-15}$

\section{Statistical Analysis}

Nine independent observers evaluated FE and VMA (standing and lying) diagnostic images for each of the 102 patients resulted in a mean and standard deviation of intervertebral rotations across 5 vertebral levels. The standard deviation represented the level of measurement variability. A comparative analysis in asymptomatic patients of $\mathrm{FE}$ between published measures of intervertebral rotation ${ }^{21}$ and the current study data performed to ensure that there were no identifiable differences. There was a significant dif-

\begin{tabular}{|c|c|c|c|c|c|c|}
\hline & \multicolumn{2}{|c|}{$\begin{array}{r}\text { Asymptomatic } \\
(\mathrm{n}=57)\end{array}$} & \multicolumn{2}{|c|}{$\begin{array}{r}\text { Symptomatic } \\
(\mathrm{n}=52)\end{array}$} & \multicolumn{2}{|c|}{ Difference } \\
\hline & Mean & Std Dev & Mean & Std Dev & Mean & $\begin{array}{r}\text { Std } \\
\text { Dev }\end{array}$ \\
\hline Age (yrs) & 48 & 11 & 53 & 13 & 5 & 2 \\
\hline Height( in) & 65.6 & 6.1 & 63.8 & 6.4 & 1.8 & 0.3 \\
\hline Weight (lbs) & 168 & 29 & 176 & 32 & 8 & 3 \\
\hline $\begin{array}{l}\text { BMI (lbs/ } \\
\left.\text { in }^{2}\right)\end{array}$ & 26.6 & 4.7 & 30.2 & 4.9 & 3.6 & 0.2 \\
\hline
\end{tabular}


ference in mean range of motion at L1-L2 and L5-S1; however there were no significant difference in the standard deviations across levels (Table 2).

To examine the inter-patient variability between bending modes within FE and VMA analysis of variance (ANOVA) was conducted for the dependent intervertebral rotation mean and standard deviations variables. Independent variables were evaluated using a $3 \times 5$ design (bending radiograghs [FE v. VMA (standing) v. VMA (lying)]) $\mathrm{x}$ (vertebral level [L1-L2 v. L2-L3 v. L3-L4 v. L4-L5 v. L5-S1] separately for the flexion/extension and left/right lateral bending modes across asymptomatic and symptomatic patients. We extended the analysis by stratifying patients into age and gender groups (18-35, 36-45,
46-55, 56+) within both patient groups. Furthermore, statistical and practical differences were studied using a post-hoc comparison (paired sample t-test) for a univariate comparison to determine where the differences occur. For all analyses, a significance level of $\mathrm{p}=.05$ was set. Data were analyzed using SAS (SAS Institute Inc, Cary, NC).

\section{Results}

Measurement variability of intervertebral rotation Analysis revealed significant measurement variability between FE and both VMA (standing and lying). Additionally, significant measurement variability was identified between $\mathrm{FE}$ and both VMA positioning under flexion/extension and left/right lateral bend-

Table 2. Inter-patient measurement variability of intervertebral rotation between standard bending radiograghs (FE) and continuous controlled bending radiograghs $(\mathrm{VMA})$ in a flexion/extension bending mode. Panel $\mathrm{A}$ and $\mathrm{B}$ reports results of the asymptomatic and symptomatic study population, respectively. ${ }^{*} *{ }^{\mathrm{p}}<0.01$, $* * p<0.05, * p<0.1$.

Panel A. Columns I-III report means and standard deviations of intervertebral rotation by level in asymptomatic individuals; columns IV-V report the differences in means and standard deviations of intervertebral rotation by level.

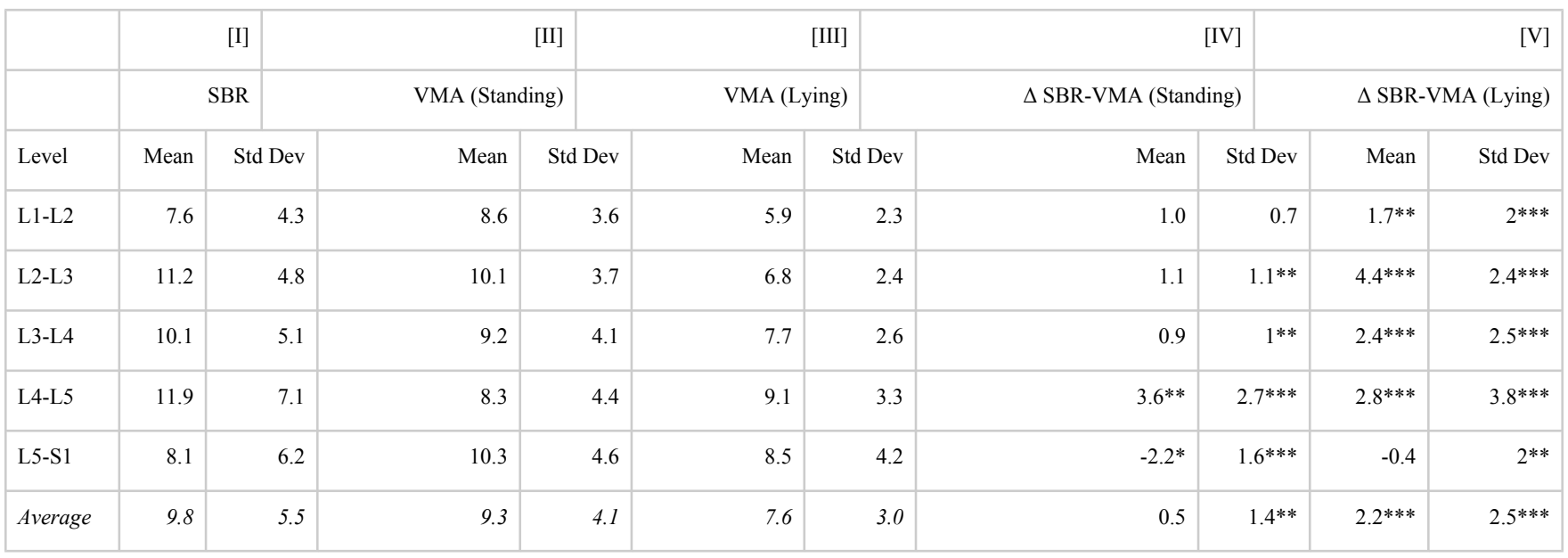

Panel B. Columns I-III report means and standard deviations of intervertebral rotation by level in symptomatic individuals; columns IV-V report the differences in means and standard deviations of intervertebral rotation by level.

\begin{tabular}{|c|c|c|c|c|c|c|c|c|c|c|}
\hline & & {$[\mathrm{I}]$} & & [II] & & [III] & \multicolumn{3}{|c|}{$[\mathrm{IV}]$} & {$[\mathrm{V}]$} \\
\hline & \multicolumn{2}{|r|}{ SBR } & \multicolumn{2}{|c|}{ VMA (Standing) } & \multicolumn{2}{|c|}{ VMA (Lying) } & \multicolumn{3}{|c|}{$\Delta$ SBR-VMA (Standing) } & $\Delta$ SBR-VMA (Lying) \\
\hline Level & Mean & Std Dev & Mean & Std Dev & Mean & Std Dev & Mean & Std Dev & Mean & Std Dev \\
\hline L1-L2 & 6.7 & 4.8 & 6.4 & 3.3 & 5.4 & 2.4 & 0.3 & $1.5^{* *}$ & $1.3 *$ & $2.4 * * *$ \\
\hline L2-L3 & 7.1 & 5.0 & 8.9 & 3.1 & 6.2 & 2.3 & $-1.8^{*}$ & $1.9^{* * *}$ & 0.9 & $2.7 * * *$ \\
\hline L3-L4 & 7.6 & 5.5 & 10.0 & 3.7 & 6.9 & 2.5 & $-2.4^{*}$ & $1.7^{* *}$ & 0.7 & $3.0 * * *$ \\
\hline L4-L5 & 6.8 & 6.2 & 9.3 & 3.0 & 8.7 & 3.1 & $-2.5 * *$ & $3.2 * * *$ & $-1.9 *$ & $3.1 * * *$ \\
\hline L5-S1 & 7.4 & 4.7 & 3.8 & 2.1 & 7.9 & 4.3 & $3.6^{* *}$ & $2.6^{* * *}$ & -0.5 & 0.5 \\
\hline Average & 7.1 & 5.2 & 7.7 & 3.1 & 7.0 & 2.9 & -0.6 & $2.2 * * *$ & 0.1 & $2.3^{* * *}$ \\
\hline
\end{tabular}

Downloaded from http://ijssurgery.com/ by guest on April 25, 2023 
ing modes. These findings are quantitatively and statistically similar across asymptomatic and symptomatic patients. Table 2, panel A reports measurement variability of intervertebral rotation between $\mathrm{FE}$ and VMA in a flexion/extension bending mode and reports the differences in mean and standard deviations in intervertebral rotation. Table 3 (panel A \& B) reports results between FE and VMA in a left/ right lateral bending mode.

Percent change in inter-patient measurement variability of intervertebral rotation

Table 4 and Table 5 report the percent change in measurement variability of intervertebral rotation between FE and VMA. Table 4, panel A providesresults for asymptomatic patients and reports that for flexion/extension bending in the standing position there is a $26 \%$ reduction in variability in VMA compared to FE. Additionally, when comparing VMA in a lying position to $\mathrm{FE}$, there is a $46 \%$ reduction in variability. Similar findings are reported in panel B which reports left/right lateral bending; indicating that in a reduction in variability of $26 \%$ and $33 \%$ is seen between standing and lying VMA to FE, respectively.

Table 5, panel A provides results for symptomatic patients and reports that for flexion/extension bending in the standing position there is a $41 \%$ reduction in variability in VMA compared to FE. Additionally, when comparing VMA in a lying position to $\mathrm{FE}$, there is a $44 \%$ reduction in variability. Similar find-

Table 3. Inter-patient measurement variability of intervertebral rotation between standard bending radiograghs (FE) and continuous controlled bending radiograghs (VMA) in a left/right lateral bending mode.

Panel A and B reports results of the asymptomatic and symptomatic study population, respectively. $* * *_{p}<0.01, * *_{p}<0.05, * p<0.1$

Panel A. Columns I-III report means and standard deviations of intervertebral rotation by level in asymptomatic individuals; columns IV-V report the differences in means and standard deviations of intervertebral rotation by level.

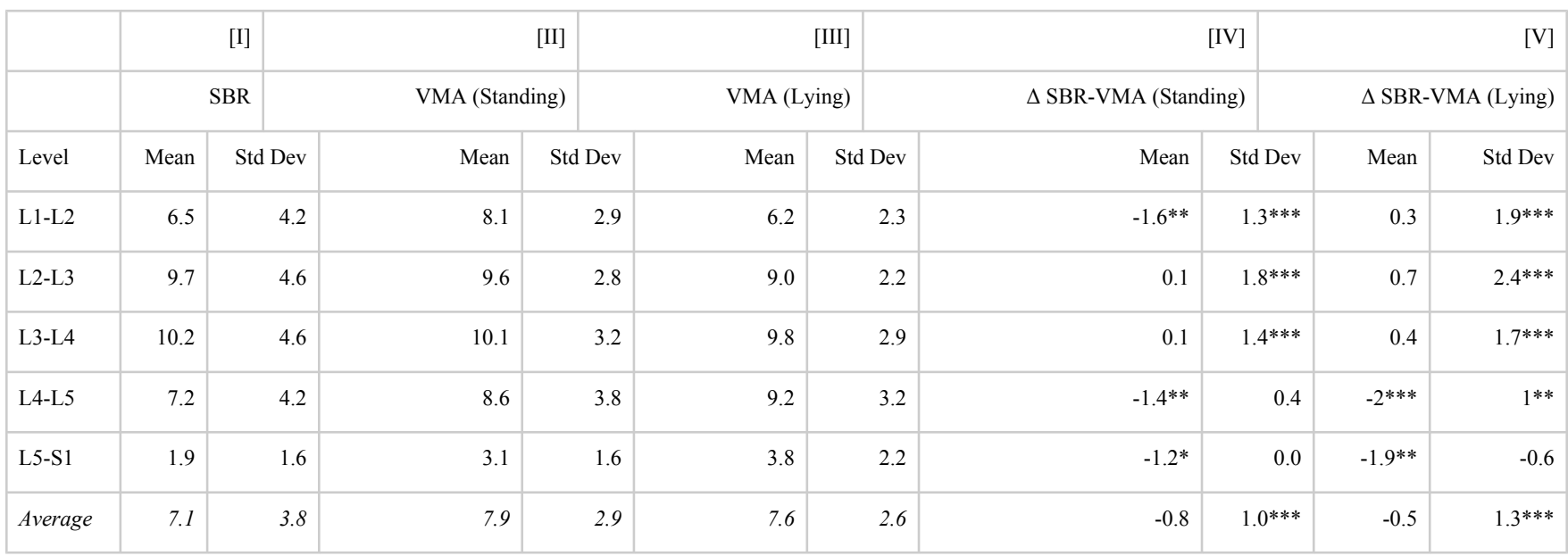

Panel B. Columns I-III report means and standard deviations of intervertebral rotation by level in symptomatic individuals; columns IV-V report the differences in means and standard deviations of intervertebral rotation by level.

\begin{tabular}{|c|c|c|c|c|c|c|c|c|c|c|}
\hline \multirow[b]{3}{*}{ Level } & \multirow{2}{*}{\multicolumn{2}{|c|}{$\begin{array}{r}{[\mathrm{I}]} \\
\text { SBR }\end{array}$}} & \multirow{2}{*}{\multicolumn{2}{|c|}{$\begin{array}{r}{[\mathrm{II}]} \\
\text { VMA (Standing) }\end{array}$}} & \multirow{2}{*}{\multicolumn{2}{|c|}{$\begin{array}{r}\text { [III] } \\
\text { VMA (Lying) }\end{array}$}} & \multirow{2}{*}{\multicolumn{2}{|c|}{$\Delta$ SIV] }} & \multirow{2}{*}{\multicolumn{2}{|c|}{$\begin{array}{r}{[\mathrm{V}]} \\
\Delta \text { SBR-VMA (Lying) }\end{array}$}} \\
\hline & & & & & & & & & & \\
\hline & Mean & Std Dev & Mean & Std Dev & Mean & Std Dev & Mean & Std Dev & Mean & Std Dev \\
\hline L1-L2 & 5.7 & 3.8 & 8.0 & 3.0 & 7.0 & 2.4 & $-2.3 * *$ & $0.8^{*}$ & $-1.3 *$ & $1.4^{* *}$ \\
\hline L2-L3 & 7.8 & 4.1 & 9.7 & 2.8 & 8.4 & 2.7 & $-1.9^{*}$ & $1.3 * *$ & -0.6 & $1.4^{* *}$ \\
\hline L3-L4 & 9.4 & 4.7 & 10.4 & 3.2 & 9.1 & 3.1 & -1.0 & $1.5^{* *}$ & 0.3 & $1.6^{* * *}$ \\
\hline L4-L5 & 6.9 & 3.8 & 8.5 & 3.8 & 8.2 & 2.5 & $-1.6^{*}$ & 0.0 & $-1.3 *$ & $1.3^{* *}$ \\
\hline L5-S1 & 2.4 & 1.3 & 2.9 & 1.4 & 3.9 & 2.8 & -0.5 & -0.1 & $-1.5^{*}$ & $1.5^{* *}$ \\
\hline Average & 6.4 & 3.5 & 7.9 & 2.8 & 7.3 & 2.7 & $-1.5^{*}$ & $0.7^{*}$ & -0.9 & $1.4^{* *}$ \\
\hline
\end{tabular}

Downloaded from http://ijssurgery.com/ by guest on April 25, 2023 
ings are reported in panel B which reports left/right lateral bending; indicating that in a reduction in variability of $26 \%$ and $23 \%$ is seen between standing and lying VMA to FE, respectively.

Measurement variability stratified by age and gender Figure 1, panels A-D report mobility by level across age. The current findings show significant correlations between a decrease in intervertebral rotation range of motion and age. More importantly as it relates to our analysis measurement variability is not statistically correlated to age (Figure 2, panels A-D). While not graphically presented, the results for gender are consistent with age indicate no significant correlation between gender and inter-patient variability. The aforementioned results are consistent across both standing and lying VMA under both flexion/extension and left/right lateral bending modes.

\section{Discussion}

The findings of the current study report significant

\begin{tabular}{|c|c|c|c|c|}
\hline \multirow[b]{2}{*}{ Level } & \multicolumn{2}{|c|}{$\%$ Change: VMA (Standing) $/ S B R$} & \multicolumn{2}{|c|}{$\%$ Change: VMA (Lying)/SBR } \\
\hline & Mean & Std Dev & Mean & Std Dev \\
\hline L1-L2 & $13 \%$ & $-16 \%$ & $-22 \%$ & $-47 \%$ \\
\hline L2-L3 & $-10 \%$ & $-23 \%$ & $-39 \%$ & $-50 \%$ \\
\hline L3-L4 & $-9 \%$ & $-20 \%$ & $-24 \%$ & $-49 \%$ \\
\hline L4-L5 & $-30 \%$ & $-38 \%$ & $-24 \%$ & $-54 \%$ \\
\hline L5-S1 & $27 \%$ & $-26 \%$ & $5 \%$ & $-32 \%$ \\
\hline Average & $-5 \%$ & $-26 \%$ & $-22 \%$ & $-46 \%$ \\
\hline \multicolumn{5}{|c|}{ Panel B. \% change in left/right lateral bending } \\
\hline & \multicolumn{2}{|c|}{$\%$ Change: VMA (Standing) $/ S B R$} & \multicolumn{2}{|c|}{$\%$ Change: VMA (Lying)/SBR } \\
\hline Level & Mean & Std Dev & Mean & Std Dev \\
\hline L1-L2 & $25 \%$ & $-31 \%$ & $-5 \%$ & $-45 \%$ \\
\hline L2-L3 & $-1 \%$ & $-39 \%$ & $-7 \%$ & $-52 \%$ \\
\hline L3-L4 & $-1 \%$ & $-30 \%$ & $-4 \%$ & $-37 \%$ \\
\hline L4-L5 & $19 \%$ & $-10 \%$ & $28 \%$ & $-24 \%$ \\
\hline L5-S1 & $63 \%$ & $0 \%$ & $100 \%$ & $38 \%$ \\
\hline Average & $11 \%$ & $-26 \%$ & $7 \%$ & $-33 \%$ \\
\hline
\end{tabular}

differences in measurement variability of lumbar spine motion between FE and VMA. The impact between diagnostics for measuring the functional characteristics of a patient's lumbar segment is essential to assessing pathologies afflicting the spine. There have been two major advancements in addressing measurement variability in $\mathrm{FE}$ in recent years. ${ }^{6-14}$ First, computer software image processing improves the precision and reliability of measurements by reducing user interaction; which likely results in less measurement variability. Secondly, the development of VMA which assesses intervertebral motion with the use of a handling device that assists patients through a standard arc of bending at a consistent rate and position. As established by the significant reduction in variability in both the standing and lying positions with VMA, the ability to accurately diagnose instability of the spine is dramatically increased.

As with any quantitative diagnostic measurement, measurement variability is a primary factor of diag-

\begin{tabular}{l} 
Table 5. Percent change in inter-patient measurement variability of \\
intervertebral rotation in symptomatic patientsbetween standard bending \\
radiograghs (FE) and continuous controlled bending radiograghs (VMA). \\
Panel A. \% change in flexion/extension bending. \\
\hline
\end{tabular}


nostic value in to distinguishing between the various types of patient conditions. ${ }^{21}$ As such, it would be plausible to infer measurement variability is the antagonist of effective diagnosis, as an increase in the measurement variability, there would be a decrease in the effectiveness of the resulting diagnosis. When we consider the current standard of care conducting functional testing of the spine involves capturing $\mathrm{FE}$ as the patient bends at a self-selected rate and position, the images captured introduces variability (by definition) because patients bend in a highly variable way. As expected, the resulting measurements taken from the FE images are highly variable, both within patients over time, and between patients.

Today's method for conducting functional testing of the spine has been widely incorporated in clinical evaluation of spine pathologies and low back pain for nearly 75 years. ${ }^{3}$ Interestingly though, given the significant advancements in functional testing protocols in other organ systems (i.e. cardiovascular), little has changed in the modalities for functional evaluation of the spine. ${ }^{2,3}$ Given the incomplete and/or ineffective imaging data, the clinician is often limited in making a definitive diagnosis.

In evaluating results of normative intervertebral motion across multiple diagnostic sites, we identified an average inter-subject variability (average SD across all levels at each site) of intervertebral motion across several studies. Pearcy and Tibrewal (1984), Boden et al (1989), and Frobin et al (1996) reported intervertebral angles for different populations studied at each respective investigational site. In aggregate their findings resulted in approximate SD of 5.2 degree of motion. Our analysis and findings made a direct comparison of intervertebral motion in the study population to that of the aggregate population, which resulted in no significant differences in measurement variability across vertebral levels. ${ }^{22-24}$ These findings would suggest the current study population is a representative patient population to previously evaluated populations.

As the cause of LBP is often unclear and up to $85 \%$ of patients with low back pain cannot be given a definitive diagnosis, epidemiological evidence would suggest diagnostic inefficiencies. ${ }^{1,2}$ Our study quantified the measurement variability between FE and VMA (standing and lying) for both asymptomatic and symptomatic patients. The findings showed a significant difference in measurement variability between FE and VMA (standing and lying) across levels and in aggregate. Measurement variability was significant greater at each level and in aggregate in FE compared to VMA in both standing and lying positions. The findings were quantitative and statistically similar for flexion/extension and lateral (left/right) bending modes. Additionally, the findings were consistent across FE and VMA in patients (symptomatic and asymptomatic); with no difference seen across patients in VMA. These findings are compelling as a means to suggest the integration of VMA as a functional evaluation modality reduces measurement variability in functional spine evaluations. Moreover, this reduction in measurement variability could be seen as an opportunity to move towards addressing the inefficiencies in functional spine diagnostics.

Spinal instability is the most common primary diagnosis for fusion..$^{21}$ There are approximately 4 million FE per year ordered in US, as the primary diagnostic tool for detecting spinal instability. Our results indicate that there are significant differences in measurement variability between the "standard of care" FE and VMA. The current results report a reduction in measurement variability between $20 \%$ and $46 \%$ depending on the patient (symptomatic and asymptomatic) and bending mode. This increased measurement variability presents the potential for diagnostic inefficiencies. Measurement variability can result in increased rates of inconclusiveness, increased rates of misclassifications in diagnostic outcomes; which presents challenges to the clinician relying on the imaging results for a definitive diagnosis. FE are ubiquitous today because it provides important information and until recent developments in VMA there have been few alternatives. The findings of the current study provide evidence that VMA can play a role in addressing the inefficiencies in the current standard of care FE.

Measurement variability in diagnostic imaging is obviously not a singular influence challenging clinician during diagnosis around low back pain. Patient specific demographics (age, gender, anthropometry, 
etc.), health history, and other may contribute to the presentation of low back pain. Although in many studies, LBP were more commonly reported in women than in men, ${ }^{25,26}$ this was not always the case in other studies or in the same studies when different definitions of pain were used. ${ }^{27}$ The same confusion exists in relation to age. Low back pain has been noted to increase with age,${ }^{27}$ while others have suggested no change over time..$^{25}$ In other cases it was found to peak in the middle years, ${ }^{27}$ and in yet others to remain the same across all ages or to diminish with age. ${ }^{25}$ Our results indicated range of motion, as measured in VMA decreased with age. Moreover while mobility decreased with age, measurement variability was consistent over time suggesting measurement variability is unrelated to age. This finding was consistent with gender as well showing no statistical difference between males and females when spine motion was measured in VMA.

To draw an analogy to another discipline, the ability to characterize a patients' endocrine system function, namely the robustness of mineralization and subsequently bone metabolism, has made DEXA (Dual Energy Xray Absorptiometry) ubiquitous. Bone mineral density is widely understood to decrease with age and exhibit differences in gender and race. As a criterion of assessment for DEXA, the World Health Organization and others utilize the Tscores and Z-scores to categorize patients based on age stratification from normal to severely osteoporotic. This dynamic categorization based on a functional DEXA exam provides clinicians a definitive and direct comparison of individuals in evaluating treatment options. Given the standardized methodology employed in DEXA studies and consistency in the imaging techniques, clinicians have a reliable test to incorporate across a wide spectrum of populations that provides age appropriate results with consistent outcomes. The spine clinician does not enjoy a functional comparative test and diagnostic outcome to make such population based comparisons from accurate age matched measurements in addressing nonspecific back pain or lumbar instability. The VMA provide much the same diagnostic consistency as the DEXA which could result in the population of a similar normative database over time.

\section{Conclusion}

Standard bending radiographs are widely considered the current standard of care for detecting lumbar instability and diagnosis the factors associated with LBP. However, inherent to the protocol for FE, there is increased measurement variability when relying on the diagnostic imaging for diagnosis. This measurement variability was reduced when including VMA into the continuum of care as a diagnostic modality. For intervertebral motion measurement variability was consistently higher in FE than VMA for both symptomatic and asymptomatic patients, across bending modes (flexion/extension and lateral bending), and in standing and lying positions. These findings suggest that the use of the VMA would result in an overall net benefit to patients receiving definitive diagnoses for instability based on intervertebral rotation.

\section{References}

1. Cyress, B. Characteristics of physician visits for back symptoms: A national perspective. American Journal of Public Health 1986; 73: 49-53

2. Finch, P. Spinal pain- An Australian perspective. Proceedings of the $13^{\text {th }}$ World Congress of the International Federation of Physical Medicine and Rehabilitation 1999, Washington DC, 243-246.

3. Knutsson F. The instability associated with disc degeneration in the lumbar spine. Acta Radiology 1944; 25: 593-608.

4. Panjabi M, Chang D, Dvorak J. An analysis of errors in kinematics parameters associated with in vivo functional radiographs. Spine 1992; 17: 200-205.

5. Dvorak J, Panjabi M, Noventoy J, Chang D, Grob D. Clinical validation of functional flexion-extension roentgenograms of the lumbar spine. Spine 1991; 16: 943-950.

6. Diab K, Sevastik J, Hedlund R, et al. Accuracy and applicability of measurement of the scoliotic angle at the frontal plane by Cobb's method, by Ferguson's method and by a new method. Eur Spine J 1995;4:291-295.

7. De Carvalho A, Vialle R, Thomsen L, et al. Reliability analysis for manual measurement of coronal plane deformity in adolescent scoliosis. Are 30 x 90 
$\mathrm{cm}$ plain films better than digitized small films? Eur Spine J 2007;16:1615-1620.

8. Tanure M, Pinheiro A, Oliveira A. Reliability assessment of Cobb angle measurements using manual and digital methods. Spine J 2010; 10:769-774.

9. Cheung J, Wever D, Veldhuizen A, et al. The reliability of quantitative analysis on digital images of the scoliotic spine. Eur Spine J 2002; 11:535-542. 10. Stokes IAF, Aronsson DD. Computer-assisted algorithms improve reliability of King classification and Cobb angle measurement of scoliosis. Spine 2006;31:665-670.

11. Gstoettner M, Sekyra K, Walochnik N, et al. Inter- and intraobserver reliability assessment of the Cobb angle: manual versus digital measurement tools. Eur Spine J 2007;16:1587-1592.

12. Shea KG, Stevens PM, Nelson M, et al. A comparison of manual versus computer-assisted radiographic measurement: intraobserver measurement variability for Cobb angles. Spine 1998;23:551-555. 13. Carman DL, Browne RH, Birch JG. Measurement of scoliosis and kyphosis radiographs: intraobserver and interobserver variation. J Bone Jt Surg Am 1990;72:328-333.

14. Mok JM, Berven SH, Diab M, et al. Comparison of observer variation in conventional and three digital radiographic methods used in the evaluation of patients with adolescent idiopathic scoliosis. Spine 2008;33:681-686.

15. Davis, R, Lee, D, Wade, C, Cheng, B. Measurement performance of a computer assisted vertebral motion analysis system. International Journal of Spine Surgery, August, 2015.

16. Panjabi M, Chang D, Dvorak J. An analysis of errors in kinematics parameters associated with in vivo functional radiographs. Spine 1992; 17: 200-205.

17. Dvorak J, Panjabi M, Noventoy J, Chang D, Grob D. Clinical validation of functional flexionextension roentgenograms of the lumbar spine. Spine 1991; 16: 943-950.

18. Breen A. A digital processing method for spine kinematics-preliminary studies. Clinical Biomechanics 1989; 3: 5-10.

19. Breen A, Muggleton J, Mellor F. An objective spinal motion imaging assessment (OSMIA): Reliability, accuracy and exposure data. BMC Musculoskeletal Disorders 2006; 7: 1-10.
20. Muggleton J, Kondracki M, Allen R. Spinal fusion for lumbar instability: Does it have a scientific basis? J Spinal Dis 2000; 13: 200-204.

21. Pearcy M, Tibrewal S. Axial rotation and lateral bending in the normal lumbar Spine measured by three-dimensional radiography. Spine1984; 9:547-661.

22. Boden S, Goodenough D, Stockham C, Jacobs E. Precise measurement of vertebral bone density using computed tomography without the use of an external reference phantom.Journal of Digital Imaging 1989; 2:31-38.

23. Frobin W, Brinckmann P, Leivseth G, Biggemann $M$, Reikerås $O$. Precision measurement of segmental motion from flexion-extension radiographs of the lumbar spine. Clinical Biomechanics 1996:

11:457-465.

24. Hartvigsen J, Frederiksen H, Christensen

K.Back and neck pain inseniors - prevalence and impact. Eur Spine J2006;15:802-6.

25. Hartvigsen J, Christensen K, Frederiksen

H.Back and neck pain exhibit many common features in old age: A population based study of 4,486 Danish twins 70-102 years of age. Spine2004;29:576-80.

26. Andersson H, Ejlertsson G, Leden I, Rosenberg C.Chronic pain in a geographically defined general population: studies of differences in age, gender, social class, and pain localization. The Clinical Journal of Pain1993;9:174-82.

27. Bovim G, Schrader H, Sand T. Neck pain in the general population. Spine 1994;19:1307-9.

\section{Disclosures \& COI}

Cheng is on the Scientific Advisory Board of Ortho Kinematics. Davis is consultant for Zimmer Biomet, LDR, and Paradigm Spine. Lee is a consultant to and is on the Advisory Board of Ortho Kinematics. Lorio is a consultant for Choice Spine, owner of IP in Transformer Spine, has options in Ortho kinematics, and has acted as Principal Investigator in studies for Globus. Protsko is on the Scientific Advisory Board of Ortho Kinematics. Wade is a consultant to Ortho Kinematics.

\section{Corresponding Author}

Chip Wade, Ph.D, Auburn University, Department 
of Industrial and Systems Engineering, 3301-G Shelby Center, Auburn, AL 36849-5346. lrw0002@auburn.edu.

Published 16 May 2016.
This manuscript is generously published free of charge by ISASS, the International Society for the Advancement of Spine Surgery. Copyright @ 2016 ISASS. To see more or order reprints or permissions, see http://ijssurgery.com. 